EUROPEAN ORGANIZATION FOR NUCLEAR RESEARCH

CERN-AS/98-001

CERN-IT $/ 98 / 2$

30 mars 1998

\title{
Afficher les documents scientifiques sur le Web
}

\author{
Michel Goossens ${ }^{a)}$ et Jean-Yves Le Meur ${ }^{\mathrm{b}}$ ) \\ CERN, CH-1211 Genève 23, Suisse.
}

\begin{abstract}
Résume
Le CERN traite chaque jour un très grand nombre de documents de recherche, principalement balisés en $\mathrm{LT}_{\mathrm{E}} \mathrm{X}$ et provenant de divers serveurs Internet. Notre but est de les afficher sur le Web via le Catalogue des Preprints de la Bibliothèque du CERN sous plusieurs formes (PostScript, PDF, GIF). Nous passons en revue la procédure de conversion et décrivons nos essais de production massive pour générer de l'HTML directement à partir des sources $\mathrm{T}_{\mathrm{E}} \mathrm{X}$. Nous finissons par une discussion de quelques développements récents dans le cadre de XML (et MML) qui amélioreront le support pour les formules mathématiques dans les programmes de visualisation.
\end{abstract}

\begin{abstract}
CERN daily handles a large number of research documents, mostly marked up in $\mathrm{HAT}_{\mathrm{E}} \mathrm{X}$ and coming from many Internet servers. Our aim is to make them easily locatable on the Web with the help of the CERN Library's Preprint Catalogue in several formats (PostScript, PDF, GIF). We review the conversion procedures and give some details on some massive production trial runs to directly generate HTML from the $\mathrm{T}_{\mathrm{E}} \mathrm{X}$ sources. We conclude with a discussion of recent developments in the framework of the XML (and MML) efforts which should ease the support of mathematics formulae in Web browsers.
\end{abstract}

\section{Présenté à la dixième conférence $T_{E} X$ européenne à Saint-Malo du 29 mars - 1 avril 1998}

Publié dans les actes: Cahiers GUTenberg 28-29, pages 181-196.

a. ) IT Division, <Michel. Goossens@cern. ch>

b. ) AS Division, <Jean-Yves.Le.Meur@cern.ch> 


\section{Le catalogue des preprints du CERN \\ 1.1 Introduction}

Le catalogue des preprints est un des plus volumineux catalogues de la bibliothèque du CERN. Cette 《littérature grise », constituée d'articles soumis pour publication, utilise le même système informatique pour cataloguer que celui des livres, périodiques, vidéos, coupures de presse, etc. Les documents peuvent provenir directement du CERN ou de n'importe quel autre institut dans le monde et ils doivent être rapidement, durablement et facilement accessibles sur le World Wide Web.

L'acquisition de ces documents est devenue de plus en plus électronique ces dernières années et l'objectif est d'approcher une acquisition $100 \%$ automatisée.

Dans cette optique, nous considérons un document comme constitué des éléments suivants :

- une information bibliographique (aussi appelée 《 méta-données »);

- un texte en papier (le document complet et imprimé);

- un texte électronique (le format est variable);

- les figures électroniques (non incluses dans le texte principal).

Chacun de ces éléments suit un traitement particulier.

\subsection{Quantité et flux}

Environ deux cent mille documents de recherche sont stockés et plus de trente mille sont en format électronique. Chaque année, douze mille nouveaux documents sont ajoutés ce qui représente mille quatre cents pages par jour, ou encore une page par minute. Parmi ces documents, une grande majorité sont en $\mathrm{T}_{\mathrm{E}} \mathrm{X}$ ou $\mathrm{LAT}_{\mathrm{E}} \mathrm{X}$.

Trois flux réguliers de documents alimentent la collection des preprints :

- ceux en provenance du CERN;

- ceux en provenance de l'archivage électronique de Los Alamos;

- ceux reçus sur support papier par courrier du monde entier.

Le traitement est différent selon l'origine, mais l'objectif est toujours le même: permettre de rechercher l'information bibliographique, visualiser le texte et l'imprimer depuis toute plateforme (X-Window, PC et Mac).

\subsection{Documents du CERN}

Le traitement le plus complexe est celui appliqué aux preprints créés au CERN même! Ceci est dû d'abord à la diversité des formats utilisés au CERN (les principaux formats sont $\mathrm{T}_{\mathrm{E}} \mathrm{X}$, Word et FrameMaker), et à l'absence de toute politique concernant l'usage d'éditeurs standards. Puis, en tant que créateur du document et quelquefois son unique distributeur, nous avons la complète responsabilité de sa bonne diffusion.

L'auteur ou le responsable envoie le document source et les méta-données à l'aide d'un formulaire sur le Web au serveur des preprints. Les méta-données sont formatées pour être ajoutées à la base de données (ALICE) où certains champs sont indexés (titre, auteurs, numéro du rapport, date, résumé, etc.). Puis le document source est converti en PostScript et en PDF qui sont ensuite rendus disponibles par leur enregistrement dans la base de données. Des contrôles de qualité (au niveau du format, non du contenu) sont réalisés par les services de la bibliothèque (voir partie gauche de la figure 1).

\subsection{Documents de l'archive électronique de Los Alamos}

Il existe en physique des hautes énergies une archive centralisée à Los Alamos pour les documents de recherche, qui y sont soumis directement par les auteurs. Ces documents sont récupérés au CERN dans leur format d'origine (souvent sous forme d'archive compactée tar.gz) pour être transformés dans un format lisible par tous.

Deux procédures sont exécutées en parallèle: l'une traite via un abonnement courrier électronique (E-mail Subscription) les méta-données alors que l'autre récupère et convertit les sources (Electronic Fulltext) des documents (voir partie centrale de la figure 1). 


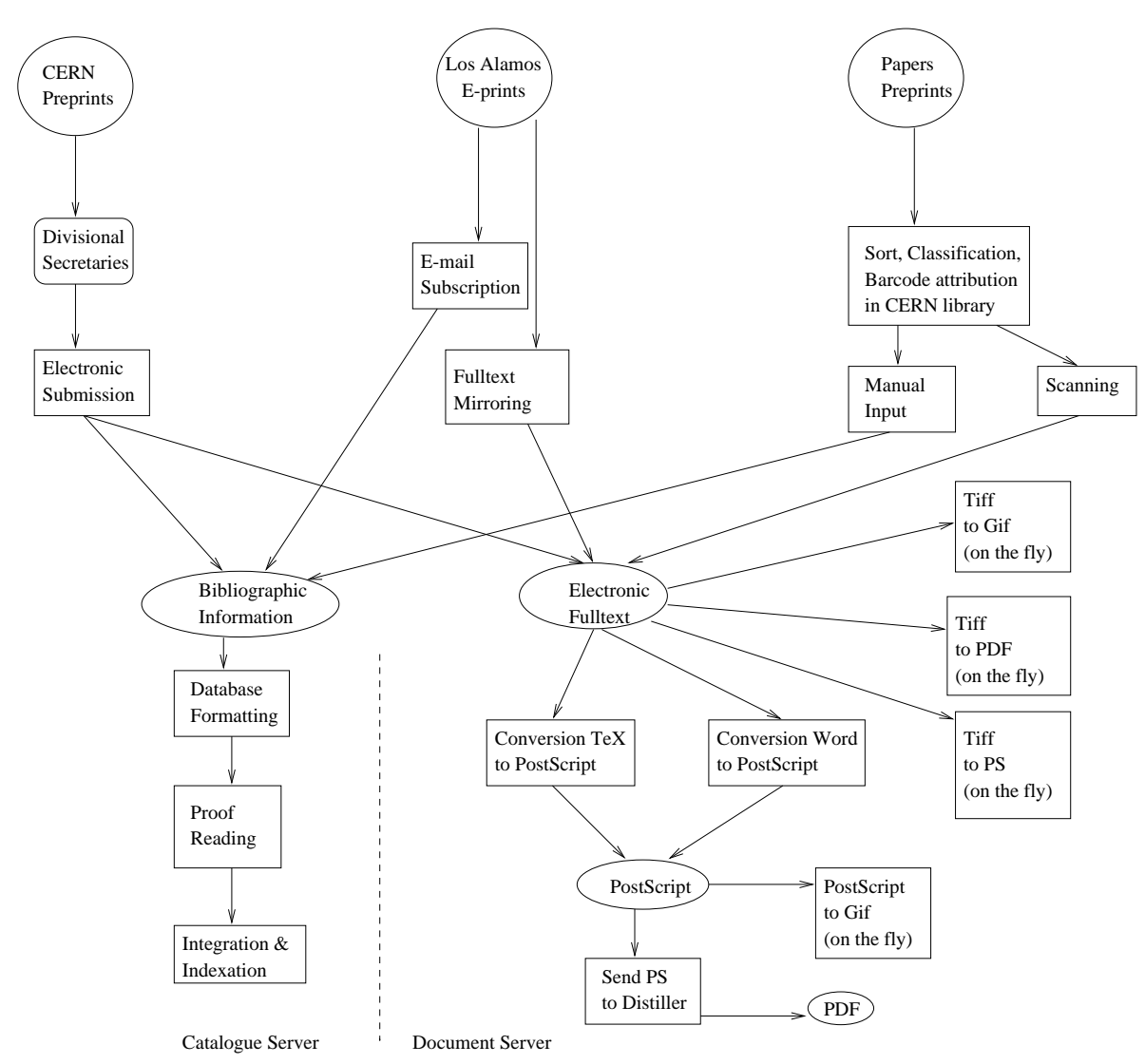

FIG. 1 - Flux des données pour l'archivage de preprints au CERN

\subsection{Documents papier}

Une quantité de plus en plus petite de documents sont envoyés par la poste à la bibliothèque centrale du CERN. Lorsqu'aucune version électronique n'est disponible et que le document est sélectionné pour diffusion, la procédure décrite dans la partie droite de la figure 1 est appliquée.

Après sélection, les documents sont digitalisés pour être conservés en format TIFF (compression G4). L'information bibliographique est entrée manuellement dans le catalogue. Plusieurs logiciels de reconnaissance optique de caractères (OCR) ont été testés afin de récupérer le résumé mais malgré des résultats assez positifs, la qualité du texte obtenu ne permet pas de l'injecter automatiquement dans la base de données. Ceci est dû en majeure partie à la présence fréquente de symboles mathématiques dans le texte, ce qui rend la reconnaissance des caractères problématique, voire impossible.

\subsection{Les conversions de formats}

\subsubsection{TEX à PostScript}

Cette procédure est bien connue. Son automatisation est réussie dans $98 \%$ des cas, l'objectif étant la création d'un document PostScript correct sans aucune intervention humaine.

La première étape identifie la version du $\mathrm{T}_{\mathrm{EX}}$ utilisée afin de lancer la conversion appropriée (tex ou latex). La conversion produit un fichier DVI.

La seconde étape transforme le format DVI en PostScript à l'aide de l'utilitaire dvips. À ce niveau, deux fichiers PostScript sont créés: le premier est compacté (gzip) et rendu disponible sur le serveur, le second est créé en incluant les polices en format Type 1 pour être transféré vers le serveur de conversion de PostScript en PDF. Cette inclusion des polices est nécessaire à la création de documents PDF de bonne qualité.

Les échecs encore rencontrés concernant cette conversion sont liés à l'usage de macros $\mathrm{T}_{\mathrm{E}} \mathrm{X}$ créées par les auteurs et que nous ne possédons pas.

Les conversions directes de $\mathrm{T}_{\mathrm{E}} \mathrm{X}$ vers PDF (pdfTeX) ou de DVI vers PDF (dvipdf) ne sont 
pas encore dans un état d'avancement suffisant pour être appliquées directement et raccourcir ainsi la procédure. Aussi créons-nous le PDF depuis le PostScript.

\subsubsection{De PostScript à PDF}

La distillation du PostScript en PDF se fait chaque douze heures en envoyant les fichiers PostScript sur un répertoire monitoré. La création des fichiers PDF est ainsi complètement automatique.

Un échec dans cette conversion signifie généralement une inconsistance dans le fichier PS, ce qui nous permet en même temps de détecter des fichiers PostScript corrompus.

\subsubsection{De Word à PostScript/PDF}

C'est au niveau de cette conversion que l'automatisation est pour l'instant la moins fiable. Une tentative a été faite de transférer vers un serveur (Mac) tout document Word nous parvenant et d'y simuler une impression pour générer le PostScript. Cela a été un échec pour deux raisons:

- les versions de Word encore en usage, sur Mac ou PC ne sont pas pleinement compatibles;

- les logiciels de dessin utilisés par les auteurs, dont les images sont ensuite « copiées/collées » dans le document Word, sont souvent inconnus ou inaccessibles sur le serveur.

Nous testons actuellement des logiciels qui permettent la conversion en traitement par lots de Word vers PostScript mais rien de vraiment satisfaisant n'est encore apparu. Le passage par le RTF (Rich Text Format) permet l'échange de l'information textuelle entre plusieurs plates-formes mais ne garantit pas l'accès aux figures.

Les documents Word représentent heureusement actuellement une minorité des documents transmis et un support manuel peut être apporté à cette tâche.

\subsubsection{Les conversions à la volée}

Afin de minimiser le stockage et la maintenance de documents en différents formats, la conversion à la volée permet d'offrir à partir d'un format unique une variété d'autres formats.

Cette technique est utilisée pour délivrer les formats suivants:

- TIFF vers PostScript: la conversion produit du PostScript niveau 2. Chaque document scanné est ainsi disponible en PostScript.

- TIFF vers PDF : le PDF créé est une image (plan de bits). Toutes les options que PDF permet (recherche de texte, copier/coller, etc) ne sont donc pas disponibles suite à cette conversion mais la visualisation et l'impression peuvent se faire via Acrobat Reader d'Adobe.

- TIFF et PostScript vers GIF : ces conversions visent à rendre possible la visualisation des documents scannés directement à l'intérieur du visualisateur (sans extension-plug-in), page par page.

\section{$2 \quad$ Le chemin direct de $\mathrm{T}_{\mathrm{E}} \mathrm{X}$ vers le Web}

Notre objectif est de permettre la lecture des preprints du catalogue CERN en HTML. La visualisation d'un document écrit en $\mathrm{T}_{\mathrm{E}} \mathrm{X}$ sur un visualisateur Web peut se faire selon plusieurs procédés (liste non exhaustive!):

- une conversion complète en HTML du document en traitement par lots (p. ex. LaTeX2HTML et TeX4ht);

- une conversion complète en HTML du document à la volée (p. ex. tth);

- une conversion partielle en traitement par lots, combinée à une application Java (p. ex. idvi);

- une lecture directe du source $\mathrm{IAT}_{\mathrm{E}} \mathrm{X}$ par une application Java (p. ex. WebEQ);

- une lecture directe du source IATEX par une application ad-hoc (p. ex. l'utilitaire techexplorer).

Pour donner une idée de la qualité de traduction nous reproduisons dans ce qui suit pour chaque programme décrit le résultat après traitement du même fragment d'un document type représentatif des formules de physique que nous avons à traiter habituellement. 


\subsection{Conversion en traitement par lots : LaTeX2HTML}

\subsubsection{Description}

LaTeX2HTML est un programme écrit en perl, qui fait appel à des utilitaires de la bibliothèque ghostscript pour la conversion des images. En général plusieurs fichiers HTML sont créés, et une table des matières permet une navigation aisée. Chaque image et certaines constructions mathématiques complexes sont converties en images GIF (ou PNG). La dernière version officielle est disponible à l'URL http://www-dsed.llnl.gov/files/programs/unix/latex2html/.

\subsubsection{Avantages}

La qualité. La qualité de l'HTML produit par LaTeX2HTML est indéniable (voir les figures 2 et 3). La mise en page et le système de navigation sont performants. Des liens sont créés vers les références. Les équations et les figures, converties en GIF, sont très lisibles. Il y a un large choix d'options pour contrôler la traduction. Pour les formules on peut les transformer «globalement » (en une partie, c'est le cas de la figure 2) ou rechercher des parties de formules qui peuvent être exprimées en HTML directement (c'est le cas de la figure 3). Il faut souligner que la consistance de la notation est très importante et que LaTeX2HTML y attache une importance extrême surtout dans le deuxième cas, où la représentation des variables à l'intérieur des images GIF et en HTML doit être compatible (p. ex. les variables doivent être en italique dans les formules et le texte). Nous remarquons toutefois quelques imprécisions dans le placement des différents sous-éléments constituant une formule, un comportement que nous retrouverons dans les approches TeX4ht et tth, qui utilisent des stratégies similaires.

Fiabilité et lisibilité. LaTeX2HTML a été largement utilisé avec succès sur des fichiers $\mathrm{T}_{\mathrm{E}} \mathrm{X}$ ou $\mathrm{LAT}_{\mathrm{E} X}$. Le résultat est très fiable. LaTeX2HTML génère des fichiers qui contiennent un HTML « normalisé », ce qui les rend lisibles par tous les programmes de visualisation. En particulier, même avec un visualisateur qui ne représente que la partie textuelle (p. ex. Lynx) on obtiendra un résultat plus qu'acceptable.

\subsubsection{Inconvénients}

Le temps. LaTeX2HTML a été intégré en test dans le cycle de production-distribution des preprints au CERN.

En moyenne, la conversion d'un article d'une dizaine de pages peut prendre plusieurs minutes temps réel (sur un poste de travail performant), ce qui présente quelques problèmes vu le nombre important de documents à traiter. À plus forte raison, cette lenteur d'exécutionprincipalement due à la conversion des images en GIF — rend également impossible son utilisation à la volée.

L'espace disque. L'obligation de collecter et de stocker les nombreux fichiers HTML ainsi que les images GIF est une lourde contrainte. La procédure requiert elle aussi un doublement voire plus, de l'espace de stockage pour un document donné.

La vitesse de chargement. Le fait que les équations soient traduites en GIF ralentit d'une façon significative la vitesse de chargement des pages riches en formules. C'est le prix à payer pour garantir l'intégrité des formules converties.

L'installation. Le script LaTeX2HTML, écrit en perl, fait appel à de nombreux utilitaires publics dont l'installation peut paraître fastidieuse, voire impossible, à un non-spécialiste (surtout sur $\mathrm{PC}$ et $\mathrm{Mac})$.

\subsection{Conversion en traitement par lots bis : TeX4ht}

\subsubsection{Description}

TeX4ht remplace les commandes $\mathrm{T}_{\mathrm{E}} \mathrm{X}$ par des séquences HTML en utilisant principalement une ou plusieurs extensions $\mathrm{IAT}_{\mathrm{E}} \mathrm{X}$. Il ne nécessite donc aucun système externe (comme perl 


$$
\begin{aligned}
& \phi_{v}\left(\lambda_{v}, \kappa, \beta^{2}\right)=\frac{1}{2 \pi i} \int_{c-i \infty}^{n+i \infty} \phi(s) e^{\lambda t} d s \quad c \geq 0 \\
& \phi(s)=\exp \left[\kappa\left(1+\beta^{2} \gamma\right)\right] \exp [\psi(\delta)] \text {, } \\
& \psi(\kappa)=\delta \ln \kappa+\left(\kappa+\beta^{2} \kappa\right)\left[\ln (\kappa / \kappa)+E_{1}(\kappa / \kappa)\right]-\kappa e^{-\kappa / \kappa},
\end{aligned}
$$

and

$$
\begin{aligned}
E_{1}(z) & =\int_{z}^{\infty} t^{-1} e^{-t} d t \quad \text { (the exponential integral) } \\
\lambda_{v} & =\kappa\left[\frac{\epsilon-\bar{\epsilon}}{\xi}-\gamma^{\prime}-\beta^{2}\right]
\end{aligned}
$$

The Vavilov parameters are simply related to the Landau parameter by $\lambda_{L}=\lambda_{v} / \kappa-\ln \kappa$. It can be shown that as $\kappa \rightarrow 0$, the distribution of the variable $\lambda_{L}$ approaches that of Landau. For $\kappa \leq 0.01$ the two

FIG. 2 - Fragment généré par LaTeX2HTML (options par défaut)

$$
\begin{aligned}
\phi_{v}\left(\lambda_{v}, \kappa, \beta^{2}\right) & =\frac{1}{2 \pi i} \int_{r-i \infty}^{r+i \infty} \phi(s) e^{\lambda s} d s c \geq 0 \\
\phi(s) & =\exp \left[\kappa\left(1+\beta^{2} \gamma\right)\right] \exp [\psi(s)] \\
\psi(s) & =\ln \kappa+\left(s+\beta^{2} \kappa\right)\left[\ln (s / \kappa)+E_{1}(s / \kappa)\right]-\kappa e^{-s / \kappa}
\end{aligned}
$$

and

$$
\begin{aligned}
E_{1}(z) & =\int_{z}^{\infty} t^{-1} e^{-t} d t \quad \text { (the exponential integral) } \\
\lambda_{v} & =\kappa\left[\frac{\epsilon-\bar{\epsilon}}{\xi}-\gamma-\beta^{2}\right]
\end{aligned}
$$

The Vavilov parameters are simply related to the Landau parameter by $\lambda_{L}=\lambda_{v} / \kappa-\ln \kappa$. It can be shown that as $\kappa \rightarrow 0$, the distribution of the variable $\lambda_{L}$ approaches that of Landau. For $\kappa \leq 0.01$ the two

Fig. 3 - Fragment généré par LaTeX2HTML (formules fractionnées) 


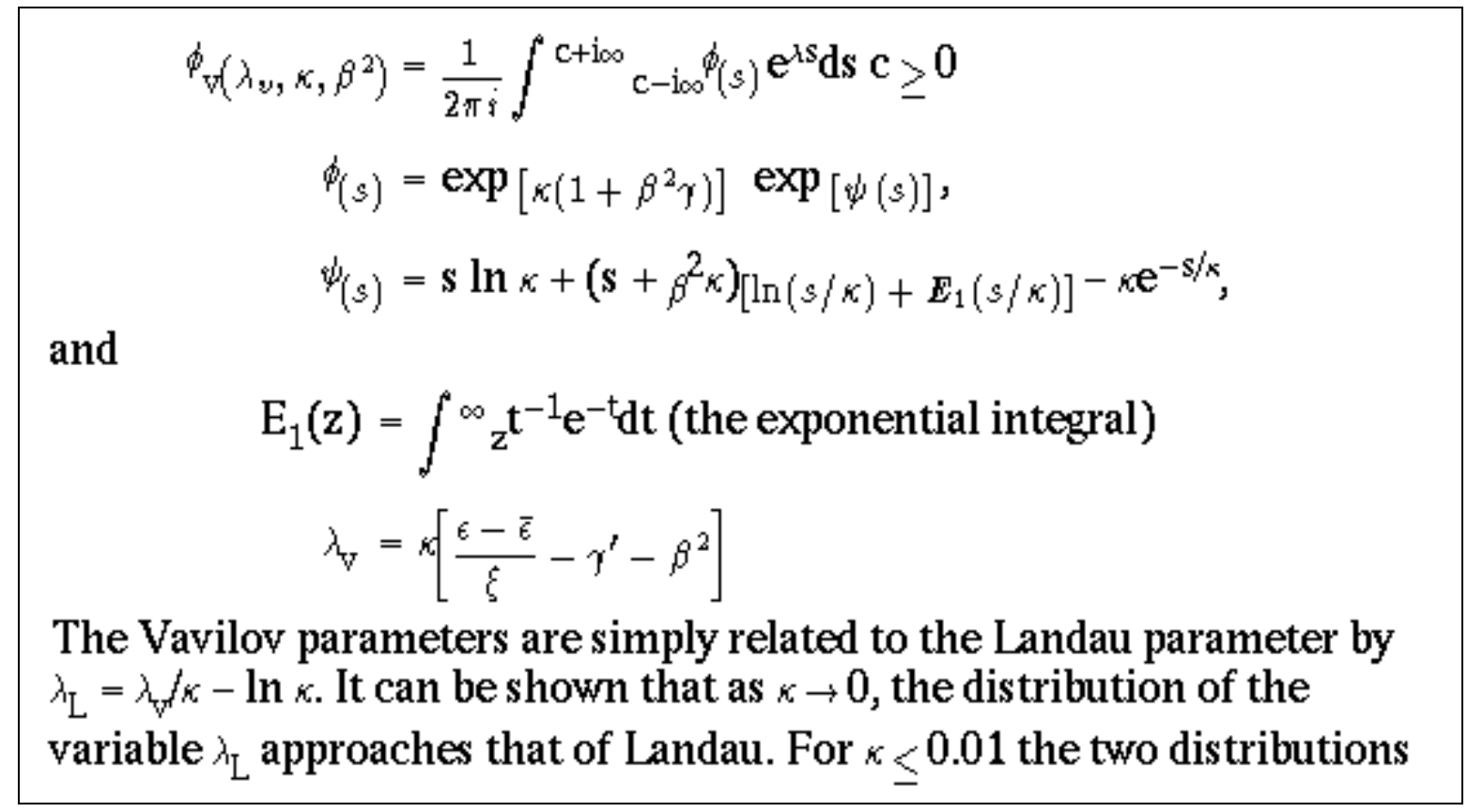

FIG. 4 - Fragment généré par TeX4ht

pour LaTeX2HTML), mais il utilise quand-même des utilitaires de la bibliothèque ghostscript pour la conversion des images et des formules mathématiques contenant des symboles spéciaux. En général il faut modifier le document source légèrement en y incluant quelques directives supplémentaires. La page de référence est à l'URL http://www.cis.ohio-state.edu/ gurari/ TeX4ht/mn.html. La figure 4 montre notre document type traité avec TeX4ht.

\subsubsection{Avantages et inconvénients}

Les avantages et inconvénients sont essentiellement ceux de LaTeX2HTML. TeX4ht est pratiquement uniquement basé sur $\mathrm{IAT}_{\mathrm{E}} \mathrm{X}$, en fait appel à des extensions (en particulier TeX4ht.sty) et des fichiers pour les polices. L'installation s'avère donc un peu plus aisée, surtout sur les machines qui ne tournent pas sous Unix. Pour les formules, TeX4ht utilise des images GIF (générées avec dvips et ghostscript). Même si, comparé à LaTeX2HTML, la qualité (notez p. ex. le placement des limites des intégrales à la figure 4) du code généré n'est pas toujours optimale, cet outil présente néanmoins une alternative intéressante.

\subsection{Conversion à la volée: tth}

\subsubsection{Description}

L'application tth est écrite en C et n'utilise aucune application extérieure pour convertir $\mathrm{T}_{\mathrm{EX}}$ en HTML. Les formules sont traduites en HTML au lieu d'être converties en images. Une description complète est disponible à l'URL http://venus.pfc.mit.edu/tth/tth.html. Le fait le plus marquant est que tth utilise les polices Symbol disponibles sur un système X-Window, Mac ou PC. Par exemple, avec X-window, il suffit d'inclure la ligne Netscape*documentFonts . charset*adobe-fontspecific: iso-8859-1 dans le fichier. Xdefaults pour utiliser les caractères en question avec Netscape. Par défaut, les images ne sont pas incluses dans le document généré mais sont disponibles comme des liens externes (il est toutefois possible de demander l'inclusion de ces images comme fichiers GIF, mais ceci complique le traitement). Le fragment du document type généré avec tth est montré en figure 5 .

\subsubsection{Avantages}

La vitesse. Le programme est d'une rapidité étonnante, à tel point que le stockage peut se faire dans un répertoire groupé (.tar) et compressé (.gz) et que la conversion (gunzip, tar xvf puis 


$$
\begin{array}{ll}
\phi_{\mathrm{V}}\left(\lambda_{\mathrm{v}}, \mathrm{k}, \beta^{2}\right) & =\frac{1}{2 \pi \mathrm{i}} \int_{\mathrm{c}-\mathrm{i}_{\infty}}^{\mathrm{c}+\mathrm{i}_{\infty}} \phi(\mathrm{s}) \mathrm{e}^{\lambda \mathrm{s}} \mathrm{ds} \mathrm{c} \geq 0 \\
\phi(\mathrm{s}) & =\exp \left[\kappa\left(1+\beta^{2} v\right)\right] \exp [\psi(\mathrm{s})], \\
\psi(\mathrm{s}) & =s \ln \kappa+\left(\mathrm{s}+\beta^{2} \kappa\right)\left[\ln (\mathrm{s} / \mathrm{k})+\mathrm{E}_{1}(\mathrm{~s} / \mathrm{k})\right]-\kappa \mathrm{e}^{-s / \kappa},
\end{array}
$$

and

$$
\begin{aligned}
& \mathrm{E}_{1}(\mathrm{z})=\int_{\mathrm{z}}^{\infty} \mathrm{t}^{-1} \mathrm{e}^{-\mathrm{t}} \mathrm{dt} \text { (the exponential integral) } \\
& \lambda_{\mathrm{v}}=\kappa\left|\frac{\varepsilon-[(\varepsilon)]}{\xi}-y^{\prime}-\beta^{2}\right|
\end{aligned}
$$

\section{The Vavilov parameters are simply related to the Landau parameter by $\lambda_{\mathrm{L}}=\lambda_{\mathrm{V}} / \mathrm{x}-\ln \mathrm{n}$. It can be shown that as $\mathrm{x} \rightarrow 0$, the distribution of the variable $\lambda_{L}$ approaches that of Landau. For $x \leq 0.01$ the two distributions}

Fig. 5 - Fragment généré par tth

tth) peut se faire à la volée sans délai significatif.

C'est un avantage considérable pour un serveur qui stocke un très grand nombre de documents.

La maintenance. La conversion à la volée permet, dans le cas où les documents sont amenés à être modifiés, de réduire à néant la maintenance de la conversion. Comparé à une procédure en traitement par lots qui demande à être relancée quand un document est changé, le gain de temps est considérable.

\subsubsection{Inconvénient majeur: la qualité}

Si certains documents apparaissent proprement, il reste fréquent que tth échoue dans la transformation de formules complexes (les commandes pour les caractères qui ne peuvent être représentés sont copiées textuellement dans la sortie HTML). Une autre faiblesse est l'absence de références croisées, ce qui est dû au fait qu'un seul passage est effectué sur le document source.

Un point supplémentaire à noter est que pour visualiser un document généré avec tth, le lecteur doit adapter l'environnement de son programme de visualisation (voir ci-dessus). Même si c'est une opération simple, un lecteur non averti (ou trop impatient) sera dans l'impossibilité de lire le document correctement.

\subsection{Conversion partielle: idvi}

\subsubsection{Description}

Le programme idvi utilise un utilitaire pour préparer un document $\mathrm{IAT}_{\mathrm{E}} \mathrm{X}$ à sa visualisation sur le Web. Une fois cette étape franchie, des applets Java permettent de lire le document exactement comme il a été formaté en $\mathrm{T}_{\mathrm{E}} \mathrm{X}$ (mêmes pages, même présentation). Une fenêtremenu, en plus de l'écran principal, permet de naviguer de page en page.

Une description complète de ce traducteur est disponible à l'adresse http://www.geom. umn.edu/java/idvi/index.html. 


$$
\begin{aligned}
& \phi_{v}\left(\lambda_{v}, \kappa, \beta^{2}\right)=\frac{1}{2 \pi i} \int_{c-i \infty}^{c+i \infty} \phi(s) e^{\lambda s} d s \quad c \geq 0 \\
& \phi(s)=\exp \left[\kappa\left(1+\beta^{2} \gamma\right)\right] \exp [\psi(s)] \text {, } \\
& \psi(s)=s \ln \kappa+\left(s+\beta^{2} \kappa\right)\left[\ln (s / \kappa)+E_{1}(s / \kappa)\right]-\kappa e^{-s / \kappa},
\end{aligned}
$$

and

$$
\begin{aligned}
E_{1}(z) & =\int_{z}^{\infty} t^{-1} e^{-t} d t \quad \text { (theexponentialintegral) } \\
\lambda_{v} & =\kappa\left[\frac{\epsilon-\bar{\epsilon}}{\xi}-\gamma^{\prime}-\beta^{2}\right]
\end{aligned}
$$

The Vavilov parameters are simply related to the Landau parameter by $\lambda_{L}=$ $\lambda_{v} / \kappa-\ln \kappa$. It can be shown that as $\kappa \rightarrow 0$, the distribution of the variable $\lambda_{L}$ approaches that of Landau. For $\kappa \leq 0.01$ the two distributions are already

FIG. 6 - Fragment généré par idvi

\subsubsection{Avantages}

Qualité et fidélité. Comme tout est basé sur le fichier DVI et traité avec les polices $\mathrm{T}_{\mathrm{E} X}$, la lisibilité du document est parfaite et les formules sont traduites de manière complètement fiable (voir figure 6). En fait idvi peut être considéré comme un visualisateur Java de DVI. Chaque page HTML est préparée séparément, et elle inclut l'appel à une classe Java.

Le résultat est complètement fidèle à la version écrite en $\mathrm{T}_{\mathrm{E}} \mathrm{X}$ par l'auteur. Le résultat s'apparente au résultat obtenu en visualisant le document en PDF avec l'utilitaire d'Adobe Acrobat Reader.

\subsubsection{Inconvénients}

Vitesse de chargement et problèmes d'impression. Comme souvent avec les applications Java, le téléchargement des classes est lent. En plus, l'interprétation du code Java nécessite des ressources en CPU non négligeables sur la machine cliente où tourne le programme de visualisation.

Un document présenté avec idvi n'est pas imprimable. Une version DVI (ou PDF) doit donc être gardée en parallèle avec les fichiers HTML propres à idvi.

Portabilité du code Java. En plus de sa lenteur, l'existence de différentes versions de Java, qui ne marchent pas toujours convenablement avec les applets générées par idvi, font que idvi ne pourra être conseillé pour la consultation massive des archives de documents. Il sera probablement supplanté bientôt par WebEQ, que nous décrirons ci-dessous.

\section{$3 \quad$ En attendant MML}

Dans un futur pas trop lointain (un ou deux ans?) nous nous attendons à ce qu'un support pour MML (Mathematical Markup Language, voir l'URL http://www .w3.org/TR/WD-math) soit intégré dans les programmes de visualisation. Ainsi les formules mathématiques seront traitées au même niveau que les parties textuelles d'un document (recherches, copier/coller, réutilisation, base de données, balises normalisées). En attendant deux approches permettent de visualiser un document $\mathrm{T}_{\mathrm{E} X}$, en le transformant en WebTeX, le langage de balisage de WebEQ, ou en traitant directement le fichier source $\mathrm{ATT}_{\mathrm{E} X} \mathrm{X}$ avec techexplorer.

\subsection{Via l'extension techexplorer}

techexplorer est conçu comme une extension (plug-in) pour les visualisateurs Netscape et Microsoft Explorer. Actuellement des versions pour Windows 95/NT, Sun et RS-6000 sont 


$$
\begin{aligned}
\phi_{\nu}\left(\lambda_{i, k} \kappa, \beta^{2}\right) & =\frac{1}{2 \pi i} \int_{c-i \infty 0}^{c+i \omega} \phi(s) e^{\lambda s} d s \quad c \geq 0 \\
\phi(s) & =\exp \left[\kappa\left(1+\beta^{2} \gamma\right)\right] \exp [\psi(s)] \\
\psi(s) & =s \ln \kappa+\left(s+\beta^{2} \kappa\right)\left[\ln (s / \kappa)+E_{1}(s / \kappa)\right]-\kappa e^{-s / \kappa}
\end{aligned}
$$

and

$$
\begin{aligned}
E_{1}(z) & =\int_{z}^{\infty} i^{-1} e^{-t} d t \quad \text { (theexponentialintegral) } \\
\lambda_{\nu} & =\kappa\left[\frac{E-\bar{E}}{\xi}-\gamma^{\prime}-\beta^{2}\right]
\end{aligned}
$$

The Vavilov parameters are simply related to the Landau parameter by

$\lambda_{L}=\lambda_{v} / \kappa-\ln \kappa$. It can be shown that as $\kappa \rightarrow 0$, the distribution of the variable $\lambda_{L}$ approaches that of Landau. For $\kappa \leq 0.01$ the two distributions are already practically

Fig. 7 - Fragment visualisé par techexplorer

disponibles et la documentation peut être consultée à URL http://www.ics.raleigh.ibm.com/ ics/techexp.htm.

techexplorer peut lire directement un fichier source $\mathrm{LAT}_{\mathrm{E} X}$, même s'il ne reconnaît pas (encore) la totalité des commandes $\mathrm{A}_{\mathrm{T}} \mathrm{EX}$. En fait, techexplorer représentera « tel quel » (en rouge) les séquences de commande $\mathrm{AT}_{\mathrm{E}} \mathrm{X}$ qu'il ne sait pas traiter directement. En plus de ces commandes $\mathrm{LT}_{\mathrm{E} X} \mathrm{X}$, techexplorer offre toute une panoplie d'extensions hypertext et multimédia, qui en font un outil performant pour préparer des documents pour le Web. La figure 7 montre le même fragment du document utilisé auparavant. Ici aucune préparation n'est nécessaire, le document source $\mathrm{ITT}_{\mathrm{E}} \mathrm{X}$ est présenté au visualisateur directement. Un support direct pour MML a été promis pour les prochains mois.

\subsection{Via Java: WebEQ}

WebEQ est un système de visualisation d'équations pour le Web utilisant Java pour rendre les formules. WebEQ a son propre éditeur pour les équations, dont l'interface de la dernière version (2.2) pour Windows NT est montrée dans la figure 8. La fenêtre en haut à gauche montre une partie des formules de notre document, à droite le menu permettant le copier-coller des symboles spéciaux, et finalement à gauche en bas, le début de la sortie MML correspondant à la fenêtre de sortie. Cet éditeur permet de composer directement les formules à l'écran ou de lire un ficher source en WebTeX, un langage qui combine HTML pour le texte et un balisage inspiré de $\mathrm{T}_{\mathrm{E}} \mathrm{X}$, mais d'une fonctionalité réduite pour les mathématiques (les formules sont saisies à la $T_{E} X$ entre $\$ \ldots$... ou $\backslash[\ldots \backslash]$ et un programme spécifique (le Sizer) les traduit en applets Java permettant la visualisation avec WebEQ.

C'est en appliquant cette procédure que nous avons obtenu le fragment de notre document type montré à la figure 9. Ainsi l'éditeur de WebEQ est un outil idéal pour s'initier à MML, comme il permet de traduire des formules (pas trop compliquées) de $\mathrm{IATEX}_{\mathrm{E}}$ en MML. Plus d'information sur WebEQ se trouve à l'URL http://www.geom.umn.edu/software/WebEQ/. 


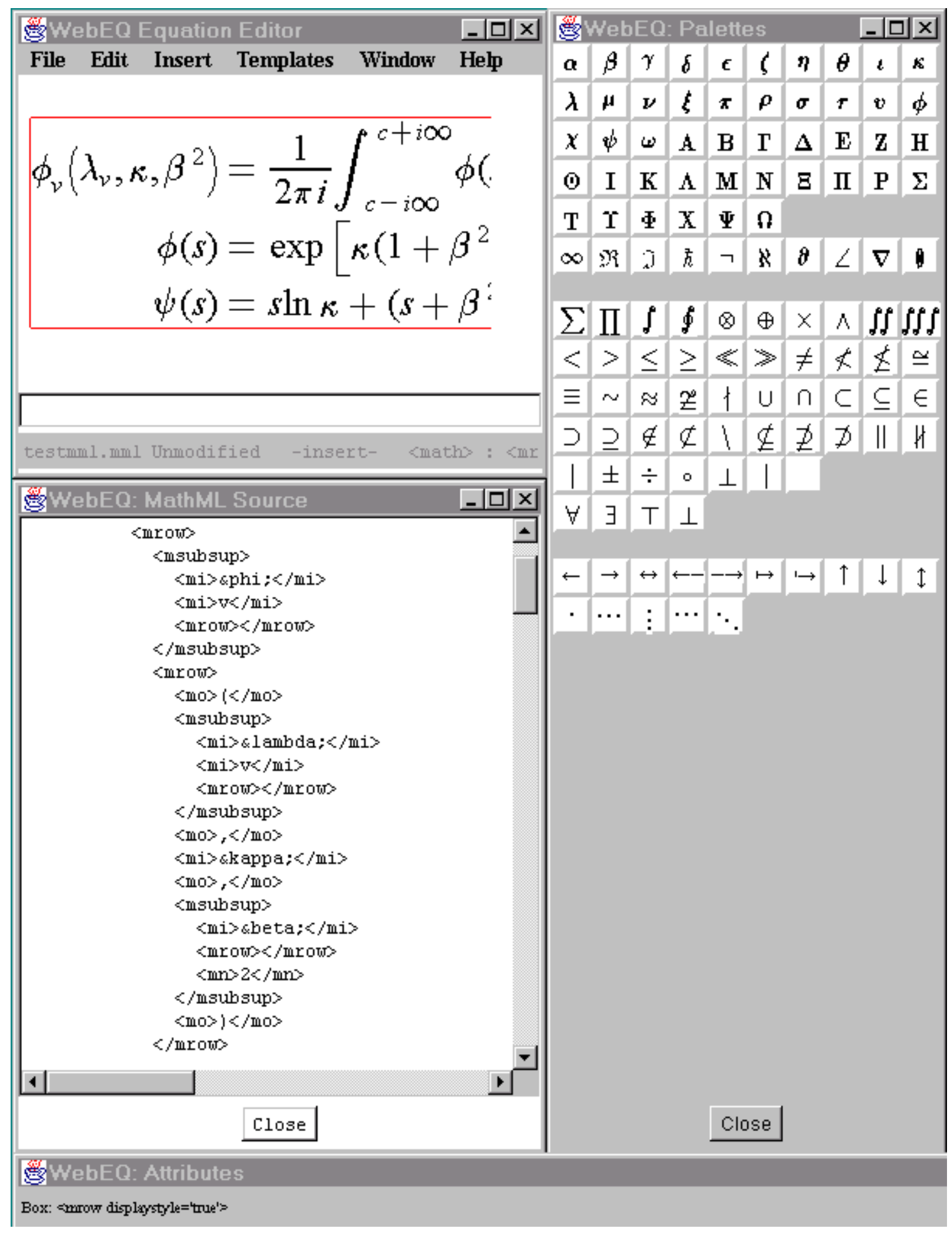

Fig. 8 - L'éditeur équations de WebEQ 


$$
\begin{aligned}
\phi_{v}\left(\lambda_{v}, \kappa, \beta^{2}\right) & =\frac{1}{2 \pi i} \int_{c-i \infty}^{c+i \infty} \phi(s) e^{\lambda s} d s \quad c \geq 0 \\
\phi(s) & =\exp \left[\kappa\left(1+\beta^{2} \gamma\right)\right] \exp [\psi(s)] \\
\psi(s) & =\sin \kappa+\left(s+\beta^{2} \kappa\right)\left[\ln (s / \kappa)+E_{1}(s / \kappa)\right]-\kappa e^{-s / \kappa},
\end{aligned}
$$

and

$$
\begin{aligned}
\dddot{E}_{1}(z) & =\int_{\underline{z}}^{\infty} t^{\cdots \cdots{ }^{1}} e^{\cdots+t^{\prime}} d t \quad \text { (the exponential integral) } \\
\lambda_{\nu} & =\kappa\left[\frac{\epsilon-\bar{\epsilon}}{\xi}-\gamma^{\prime}-\beta^{2}\right]
\end{aligned}
$$

The Vavilov parameters are simply related to the Landau parameter by $\lambda_{L}=\lambda_{v} / \kappa-\ln$. It can be shown that as $k \rightarrow \mathrm{C}$, the distribution of the variable $\lambda_{L}$ approaches that of Landau. For $\kappa \leq 0.0$. the two distributions

Fig. 9 - Fragment généré par WebEQ

\section{Conclusions}

Préparer des documents scientifiques pour les visualier sur le Web n'est pas toujours trivial, surtout quand l'information contient des parties mathématiques. Les problèmes sont maintenant bien connus (voir p. ex. à l'URL http://forum. swarthmore.edu/typesetting/index.html) et les deux seules solutions complètes sont soit une sortie PDF (ou PostScript), soit une conversion de toutes les formules en images. C'est la philosophie (plus ou moins) adoptée par presque tous les programmes qui transforment un fichier source $\mathrm{LAT}_{\mathrm{E}} \mathrm{X}$ en ces formats. Toutefois dans un cadre de production et de distribution massive de documents, la solution 100\% HTML n'est pas encore fiable.

Le développement et la mise à disposition générale de XML et MML dans les deux prochaines années assureront que les parties «formules » (mathématiques, chimie, etc.) pourront être traitées de manière plus satisfaisante par les programmes de visualisation (et les bases de données pour l'extraction de l'information). En attendant, les approches hybrides, comme celles évoquées dans cet article, permettront au plus grand nombre de profiter d'une distribution quasi-instantanée de l'information sur l'Internet. 